\title{
Sympathectomy for truncal hyperhydrosis after traumatic paraplegic injury
}

\author{
Malek G. Massad, MD, Alexander Evans, MD, Helene Rubeiz, MD, Fadi Khoury, MD, Andrew Brown, MD, \\ Janet Karol, MSN, Janeen Hernan, MSN, and Kelly Kalcevich, PA, Chicago, III
}

$\mathrm{U}$ pper thoracic (T2-T3 or T2-T4) sympathectomy with preservation of the stellate ganglion is routinely used for treatment of palmar and axillary hyperhydrosis and also for relief of advanced cutaneous vasoconstriction of the fingers and hand in patients with Raynaud disease. Thoracic sympathectomy has also been advocated and used as a last resort for patients with refractory angina pectoris and those with sustained ventricular tachyarrhythmias that are not responsive to more standard medical and surgical therapy. To our surprise, an extensive MEDLINE search of the world literature from 1966 to date showed no published data on the use of extended thoracic sympathectomy for treatment of hyperhydrosis of the torso, particularly for patients with spinal cord injuries. In this communica-

From the Division of Cardiothoracic Surgery, Department of Surgery, The University of Illinois at Chicago, Chicago, Ill.

Received for publication Feb 25, 2002; accepted for publication March 24, 2002.

Address for reprints: Malek G. Massad, MD, University of Illinois at Chicago, Division of Cardiothoracic Surgery, 840 S Wood St, CSB Suite 417 (MC 958), Chicago, IL 60612 (E-mail: mmassad@uic.edu).

J Thorac Cardiovasc Surg 2002;124:636-8

Copyright $(2002$ by The American Association for Thoracic Surgery

$0022-5223 / 2002 \$ 35.00+0 \quad \mathbf{1 2 / 5 4} / \mathbf{1 2 5 1 6 6}$

doi: $10.1067 / \mathrm{mtc} .2002 .125166$ tion we describe the surgical outcome of bilateral extended thoracic (T2-T6) sympathectomy in a man with paraplegia and hyperhydrosis of the torso.

\section{Clinical Summary}

A 25-year-old man had flaccid paralysis, anesthesia below T4, and impaired vesical and anal sphincter control that developed immediately after a fall from a tree. The fall caused T4 and T5 vertebral body fractures and necessitated internal fixation of the thoracic (T3-T6) spine and surgical stabilization with two metallic rods placed through a posterior midline approach. At the time of injury the patient had a right hemopneumothorax that required a tube thoracostomy. During the course of his illness, the patient acquired hyperhydrosis over the torso below the T4 dermatome, manifesting as excessive sweating over the chest, abdomen, and back from the level of the nipples down. The hyperhydrosis was less exaggerated over the lower extremities. It was more pronounced with minimal activity of the upper extremities and was easily triggered with exposure to a warm or hot environment. The hyperhydrosis required the patient to change his undershirt six to seven times per day, causing social inconvenience and embarrassment. His working mother, who is also his primary caregiver, accompanied him to the clinic visits and reported being overwhelmed by his care because, among other things, of her continuous need to wash and dry his undershirts. The patient had Foley catheter drainage of his urinary bladder initiated after his injury. He also underwent a 

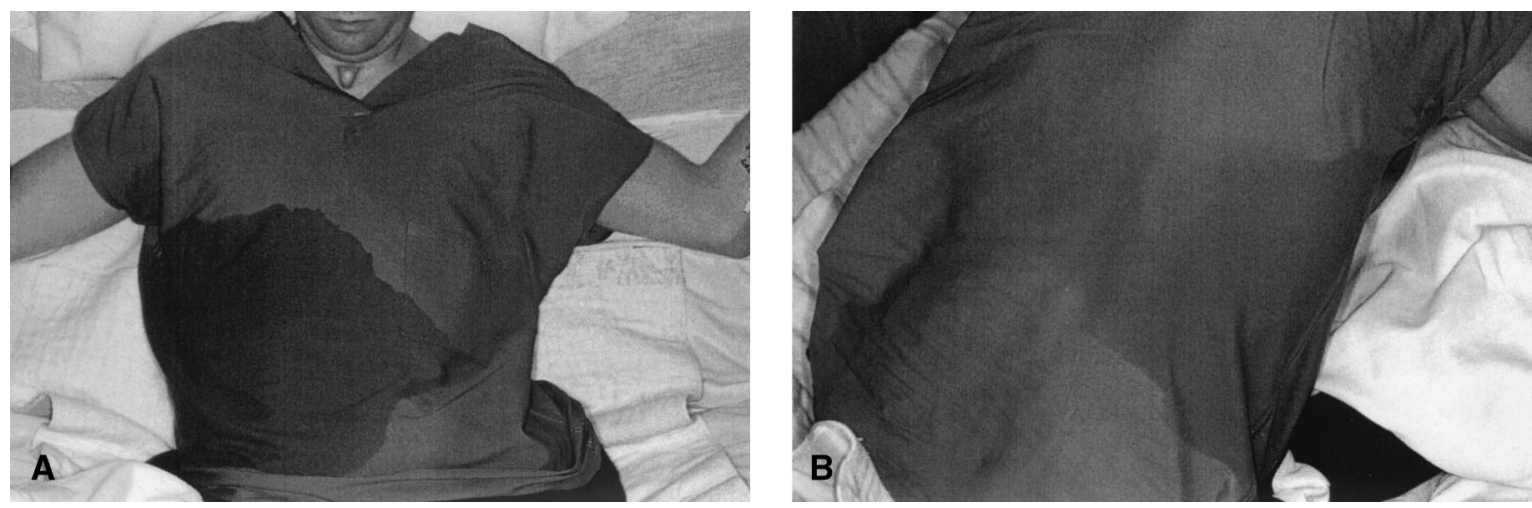

Figure 1. A, After left sympathectomy and before right sympathectomy, frontal view of patient dressed in green scrubs demonstrates absence of sweat on scrub shirt on left side of chest from nipples down and excessive sweating on right side. B, Posteroanterior view at same time demonstrates similar findings over back.

diverting colostomy that was performed because of stool incontinence.

The patient was referred to the thoracic surgery service 5 years after the initial injury by his colorectal surgeon for evaluation for surgical management of his hyperhydrosis. His medical history was significant for drug abuse and one suicide attempt at the time of injury. He has been drug free since the accident. His physical examination showed a pleasant, thin young man who used a wheelchair. He had flaccid paralysis of both of his lower extremities with severe muscle wasting. He had absence of pain, temperature, and pinprick sensation below the T4 level. His gray undershirt was soaked with sweat. His laboratory workup was not revealing. A plain radiograph of the chest was unremarkable except for the implanted metallic foreign bodies in the thoracic spine.

The patient was admitted to the hospital with the plan of performing bilateral video-assisted thoracoscopic sympathectomies in the same setting with general anesthesia. However, dense adhesions in the left pleura, conceivably related to an unrecognized hemothorax at the time of injury or later at the time of spine fixation, precluded video-assisted thoracoscopy. A plan to stage the bilateral sympathectomies was therefore adopted. The left thoracic (T2-T6) sympathectomy was performed through a muscle-sparing posterolateral thoracotomy through the fourth intercostal space. The patient was extubated in the operating room. On the same day, he noted complete cessation of sweating on the left side of his trunk but continued to have excessive sweating on the right side (mirror image). A left pleural chest tube was removed the day after his left thoracotomy, and he was discharged home on postoperative day 4.

Three weeks after discharge, he was readmitted for a right thoracic sympathectomy. Frontal and back photographs of him in bed taken before the operation, 3 hours after he was dressed in a green scrub shirt to demonstrate color contrast, are shown in Figure $1, A$ and $B$. The right thoracotomy was also performed through a muscle-sparing thoracotomy, because adhesions from his previous hemopneumothorax precluded the video-assisted thoracoscopic approach. Sympathetic denervation was confirmed by histopathologic examination of the specimen, demonstrating presence of sympathetic ganglia and nerve fibers. A right pleural chest tube was placed during the operation and was removed the next day. Physical evaluation at that time noted anhydrosis on both sides of the chest, abdomen, and back. The patient was discharged home on postoperative day 4. At a follow-up clinic visit 6 weeks after the second operation, he continued to be free of sweat over the torso, with no compensatory sweating elsewhere. He expressed relief and satisfaction with the surgical outcome.

\section{Comments}

It has been clearly demonstrated that significant differences from healthy volunteers in the thermoregulatory response to exercise are present in patients with spinal cord injuries. ${ }^{1,2}$ In healthy persons the rise in core temperature depends on the metabolic rate during exercise and is independent of the skeletal muscle mass used. This thermoregulatory response is impaired in patients with spinal cord injuries for several reasons, including loss of autonomic control for vasomotor responses, reduced thermoregulatory effector and adaptive responses for a given core temperature, and loss of skeletal muscle pump activity from the paralyzed limbs. As a result, patients with spinal cord injuries have reduced ability to tolerate thermal extremes and to perform aerobic exercise. ${ }^{2-4}$ Dawson and associates $^{5}$ have suggested that the lower limb temperature in patients with paraplegia may be greater than that in healthy persons, probably because of venous pooling in the legs.

Division of the sympathetic trunk from the second rib down to the fifth or sixth rib will interrupt all sympathetic outflow to the hands, axillae, and torso, except that which might pass through the nerves of Kunz or the intercostobrachial nerve. It is always advisable to restrict the use of electrocautery for dissection to the inferior end of the divided sympathetic trunk and to divide the sympathetic trunk on the second rib with scissors rather than diathermy to avoid burn injury to the stellate ganglion and Horner syndrome. ${ }^{6}$ Horner syndrome continues to occur in about $5 \%$ to $10 \%$ of cases after upper thoracic sympathectomy for palmar or axillary sympatholysis. 
Compensatory sweating is not uncommon after upper thoracic sympathectomy and has been observed to occur with a variable frequency. ${ }^{7}$ In a review by Andrews and Rennie ${ }^{7}$ of 42 patients who underwent bilateral T2 to T3 thoracoscopic sympathectomy for palmar or axillary hyperhydrosis, the severity of compensatory sweating was assessed with a linear analog scale. Sweating occurred in 36 patients ( $86 \%$ ). It was assessed as severe in 10 of 36 patients $(28 \%)$, as moderate in $16(44 \%)$, and as minimal in 10 $(28 \%)$.

In summary, bilateral extended thoracic sympathectomy is effective in relieving truncal hyperhydrosis of the torso in patients with paraplegia caused by spinal cord injuries. Whenever indicated, sympathectomy may be initially attempted thoracoscopically unless technical considerations, such as dense adhesions or pleural symphysis, preclude that technique. We advocate considering this operation for such patients, particularly when lessinvasive techniques, such as pharmacologic sympathetic blocks, are ineffective and when the hyperhydrosis becomes overburdening and difficult to handle.

\section{References}

1. Kneisley LW. Hyperhydrosis in paraplegia. Arch Neurol. 1977;34: 536-9.

2. Sawaka MN, Latzka WA, Pandolf KB. Temperature regulation during upper body exercise: able-bodied and spinal cord injured. Med Sci Sports Exerc. 1989;21(5 Suppl):S132-40.

3. Yamasaki M, Kim KT, Choi SW, Muraki S, Shiokawa M, Kurokawa T. Characteristics of body heat balance of paraplegics during exercise in a hot environment. J Physiol Anthropol Appl Human Sci. 2001;20: 227-32.

4. Gass EM, Gass GC. Thermoregulatory responses to repeated warm water immersion in subjects who are paraplegic. Spinal Cord. 2001; 39:149-55.

5. Dawson B, Bridle J, Lockwood RJ. Thermoregulation of paraplegic and able bodied men during prolonged exercise in hot and cool climates. Paraplegia. 1994;32:860-70.

6. Collin J. Predicting changes in the distribution of sweating following thoracoscopic sympathectomy [letter]. Br J Surg. 1998;85:570.

7. Andrews BT, Rennie JA. Predicting changes in the distribution of sweating following thoracoscopic sympathectomy. Br J Surg. 1997; 84:1702-4. 